\title{
DEVELOPING ENABLING ENVIRONMENTS IN PRACTITIONER TRAINING
}

\author{
Graham Whitehead \\ Division of Social Work and Professional Practice \\ School of Social Sciences \\ Nottingham Trent University
}

\begin{abstract}
In response to the World Health Organisation's (WHO) recommendations on effective and ethical disability provision, training providers need to consider more widely how to deliver psychological training which is sensitive to the needs of service users who have a disability. Johnson \& Haigh (2011) discuss the use of the term 'enabling environments' which usefully summarises the challenge facing the profession with regard to practitioner training. Counselling and Psychotherapy education, by its very nature, requires training providers to demonstrate a commitment to accessibility and social inclusion for people with disabilities. By modeling examples of good practice, training providers can affirm and promote competence in professional practice which consequently impacts standards of psychotherapeutic care for this population. The creation and promotion of an enabling environment in practitioner training is achieved in a number of ways: affirming basic principles of social inclusion, modeling policies and procedures which shape the training environment, demonstrating evidence of professional practice in the disability arena and the assessment of practitioner competence to practice in a transcultural environment.

Standards of ethical practice in this field warrant a clear statement and focus by training providers and a move towards the achievement of empathic resonance in practitioner training is suggested. This can only be effectively demonstrated where institutional policies and procedures are established to reflect the professional standards of the profession. The article includes several vignettes which aim to highlight consideration of the creation of an enabling environment in psychological training provision.
\end{abstract}

\section{Keywords}

Enabling Environments, Professional Practice, Disability, Transcultural, Ethical Practice, Empathic Resonance 


\section{Introduction}

The challenge of providing a psychotherapeutic training environment that responds appropriately to issues of social inclusion is one that training educators have faced in the design and delivery of the counselling and psychotherapy curriculum since training courses emerged in the mid- late twentieth century. This challenge requires a delicate balance and incorporation of the principles of social inclusion, relevant national legislation, professional codes of conduct and the development of anti-oppressive practice. Within cultures where psychotherapeutic interventions used to support an individual with mental health issues contrast with more traditional methods of treatment, for example shamanism or traditional healing practices, consideration needs to be given to the cultural appropriateness of a psychological approach. This challenge of creating an enabling environment, in psychotherapeutic education, training and practice is the major focus of this article. The term 'enabling environment' has been used by authors in many disciplines to develop the debate but there is in reality a limited and variable definition of the term. The author offers the following definition pertinent to psychotherapeutic education, training and practice:

“ an enabling environment aims to develop a psychotherapeutic milieu which takes into account the range of physical, psychological and psychosocial needs that an individual may require to fully develop their abilities and competencies".

Johnson \& Haigh (2011 p.17) discuss the use of the term 'enabling environments' which usefully summarises the challenge facing the profession with regard to practitioner training. This paper will explore the relevance and significance of 'enabling environments' applied to the context of psychotherapeutic training and will consider the position of training educators involved in the design and delivery of practitioner training. Moreover, in response to the World Health Organisation's (WHO) recommendations on effective and ethical disability provision, the paper will explore how training providers have a duty to consider more widely how to deliver psychological training which is sensitive to the needs of service users who have a disability.

\section{Context}

As psychotherapeutic training has evolved to incorporate the essence of multicultural and bicultural practice, specifically by the development of practitioner transcultural competencies McFadden(1993), Arrendondo et al (1996), the profession has in general responded appropriately to the specific requirements of cultural and social norms. The current decade dominated by economic pressures in many countries in the shape of austerity measures, has resulted in significant shifts in social and cultural attitudes, especially in the areas of disability and immigration. Joppke (2004 p.237) has highlighted what has been termed 'a retreat from multiculturalism', a theme recently widely debated and this viewpoint subsequently challenges the values that hold together many psychotherapeutic training provisions. In contrast, there are authors have commented that'across the Western democracies, we see a trend towards the increasing recognition of minority rights' Banting \& Kymlicka (2013 p.3). The same trend has evolved in psychotherapeutic training provisions in 
many non-Western countries although issues of access, equity and capacity are usefully raised by Neubauer \& Tanaka (2011) in an Asia-Pacific context which alludes to the possibility that physical accessibility still needs further consideration by some educational institutions.

This debate raises the question of whether the specific focus and development of an 'enabling environment' is in fact necessary in practitioner training. Is it preferable to assume that counselling trainees will arrive with personal and 'civic' values that will subsequently be reflected in their professional competence? On the contrary, the author believes that this is not a risk that the profession can choose to take. It is only by highlighting the relevance of practitioner competencies in psychotherapeutic training by incorporation of structured curriculum design that will ensure that practitioners deliver competence in this area, with of course the consequence that service-users receive the quality and standard of practice which meet statutory requirements reflected in professional codes of conduct.

Professor Sun in her opening address at the Asia-Pacific Rim International Counselling Conference (APRICC) 2013 commented that counselling and psychotherapy as a profession in Asia-Pacific regions is 'no longer the new kid on the block,' suggesting that western theoretical models should perhaps at this stage in the development of psychotherapeutic education sit alongside Asia-Pacific theory and practice rather than dominate it. The author fully supports such a position but contends that there are core values which permeate both Asia-Pacific and western psychotherapeutic practice. In contrast, it is worth noting the comment made by Montgomery (2013) in a discussion about the practice of counselling and psychotherapy in Malaysia, that the profession has been significantly influenced by Western approaches to theory, practice and measurement. This article made a case to develop practice based-knowledge with more regular clinical supervision and focus on personal process. It is in the spirit of this development that core competencies around disability provision can be improved.

\section{Interpretation of the recommendations of the World Health Organisation.}

The World Health Organisation's (WHO) recommendations on effective and ethical disability provision by consequence impact the need for training providers to consider more widely how to deliver psychological training which is sensitive to the needs of service users who have a disability.

Recent estimates from WHO (2011) indicate that more than one billion people in the world live with some form of disability of whom nearly 200 million experience considerable difficulties in functioning( thus indicating a potential global prevalence closer to $20 \%$.) Prevalence is also predicted to rise with the increase predominantly due to ageing populations impacted by chronic health conditions such as diabetes, cardiovascular disease, cancer and the increased diagnosis of specific mental health disorders. With such factors in mind, the WHO recently produced the World Report on Disability to provide the evidence for progressive policies and programmes to improve the lives of people with disabilities. The significance of the WHO predictions impact future service delivery planning for mental 
health provision in Europe and the Report includes specific recommendations which suggest that commissioning bodies should:

- Enable access to all mainstream systems and services

- Invest in specific programmes and services for people with disabilities

- Contribute towards a national disability strategy and plan of action

- Involve people with disabilities

- Increase public awareness and understanding

- Improve disability data collection

- Strengthen and support research on disability

(World Health Organisation 2011)

Although these recommendations are essentially focused on effective and ethical provision, they can be usefully considered and interpreted from the point of view of training and curriculum design. With specific reference to psychotherapeutic training, these recommendations can be interpreted to highlight the following aspects of practitioner training:

- Appropriate physical access to psychotherapeutic training for practitioners with a disability

- A curriculum delivered to involve educators and practitioners who have a disability which by consequence increases practitioner awareness and understanding of the relevance of competencies in this area

- Development of relevant programmes which aim to develop practitioner competence in the ability to work with service-users who have a disability.

- Evaluation of the training competencies achieved by student practitioners in the field

- Contribute towards the development of disability research themes in professional practice.

- Development of institutional policies and procedures to reflect the professional standards of the profession

\section{Appropriate access to psychotherapeutic training for practitioners with a disability.}

Accessibility to teaching and learning is central to creating an enabling environment for student practitioners with a disability - without this central tenet in situ the training environment per se will be disabling and be likely to inhibit professional progression.

Although this may appear to be a mandatory requirement for most educators, the reality is that accessibility issues frequently create an unwelcome hurdle for student practitioners. The challenge of making the learning environment physically accessible is one that most training providers have tackled due to the legal and statutory requirements of the respective national legislation. It does, however, need to be recognised that in some countries access may be viewed as a desirable yet unattainable goal given the economic and political context. The vignettes included in this article aim to interpret the WHO recommendations with specific reference to psychotherapeutic training. These are offered as food for thought as opposed to being prescriptive. 


\section{Vignette 1}

Alia is a counselling student on a Postgraduate Programme at a major Hong Kong University. She was born with a visual impairment and provides a statement from her physicians to identify her range of visual ability. Her supervisor has raised some issues in terms of how she relates to her clients in her practice placement. This partly relates to her missing important non-verbal cues from her clients. Alia has also visited her tutor to highlight how she would prefer advance notification of all teaching materials so she can prepare in advance of sessions.

This vignette raises the theme of accessibility to course materials and some of the issues that practice settings might raise for students with a disability. Accessibility can also refer to the development of teaching and learning resources which are designed to enable professional development opportunities for student practitioners who have a disability. For example, the increasing reliance on e-learning technology does pose a considerable challenge for those students with limited visual ability. Although the student may be able to access relevant elearning resources, educators need to be mindful of the additional time that such students might require and hence the release of teaching materials in a timely manner is required in order to allow time for the student to access and familiarise themselves with relevant resources. It is also worth considering making hard-copy materials in larger font regularly available at the commencement of taught sessions. This can apply, for example, to standard quality assurance procedures where questionnaires are used to illicit student feedback on the Course/Programme. This would be a useful example of developing institutional practices to create an enabling environment and can be construed as a modification to institutional practice taking into account the WHO recommendations outlined above.

\section{A curriculum delivered to involve both educators and practitioners who have a disability which by consequence increases practitioner awareness and understanding of the relevance of competencies in this area.}

The challenge for educators is to strike a balance between functional and academic curriculum content. The aim is to develop the concept of symbolic inclusion, which is a socially constructed meaning of inclusion discussed by Bentley, $\mathbf{J}$ ( in Rix et al 2010, p257) which is defined as:

' the accommodation, assimilation, appreciation and engagement of one's interaction partner' (in this instance the definition referred to working with children with severe disabilities.)

Symbolic inclusion is achieved when individuals choose to become conscious of and pay attention to one other in the professional encounter. This is to be contrasted with symbolic exclusion where individuals consciously refuse or habitually fail to accommodate, assimilate, appreciate and engage with one another. 
As a concept, symbolic inclusion can apply usefully to the psychotherapeutic training arena whereby the facilitator can contribute to the development of a curriculum (and curriculumbased activities) which accommodate, assimilate, and develop appreciation and engagement around disability-related themes. This of course applies to disabled and non-disabled students.

\section{Vignette 2}

A group of disabled-students representing the Disability Action Group of the Student's Union offer to work with academics to develop enabling environments for students of the College. In discussions with Programme Leaders, the issue of the appropriateness of some course content is questioned with a desire to re-balance the content to include less visual and more audio material.

\section{How might a psychotherapeutic training facilitator progress this ideal?}

The use of language to refer to disability is a sensitive area that warrants clear consideration by training providers. Thompson (2011) useful highlights this issue by commenting that insensitive language used to refer to people with disabilities can be construed as a form of disempowerment. He goes on to suggest that infantilizing language towards disability is often rooted in patterns of communication which are routine and is probably best viewed as a clumsy misuse of power as opposed to being deliberate. This is particularly apt in the case of trained practitioners, who in general could be deemed to have higher levels of sensitivity in linguistic communication on disability themes. In the scenario where counselling trainees have a mental health diagnosis, particular care needs to be paid to how, for example, the facilitator refers to discussion and diagnostic indications of schizophrenia which of course could be significantly sensitive for the trainee and is presented inappropriately can result in the potential for shaming the student.

\section{Vignette 3}

Samuel is in the third year of his Psychotherapy training at a university in Otago, New Zealand. He has a particular interest in how mental health diagnoses can impact a client's level of confidence, functioning and ability. His research has explored attitudes that practitioners have towards mental health diagnoses and his findings highlight how in supervision meetings diagnostic terminology is often used liberally to refer to particular client or client group.

The challenge is to strike a balance between a focus on disability themes in practitioner training without over-emphasising the specific needs of those students on the programme or clients in practice settings. There could be a danger of over-emphasis which might alienate or at least make certain individuals feel overly-exposed. This tendency naturally undermines any attempt at effective social inclusion of trainees on psychotherapeutic programmes. The vignette highlights how training providers have a strong moral and ethical position to play in how mental health diagnoses are introduced as part of the curriculum. 


\section{The development of relevant training programmes which aim to develop practitioner competencies in the ability to work with service-users who have a disability.}

The maxim that learning cannot be achieved without making mistakes is pertinent here. Psychotherapeutic training necessarily involves placement or clinical practice in which trainees build experience by a specified amount of counselling hours of client contact. Training providers hence need to consider the quality of the experience that trainees have access to. Does for example this offer the opportunity to work with clients who have learning difficulties, or physical disabilities? This subsequently raises issues for clinical practice, education and supervision. It is here the training organisations can be specific about the learning outcomes for counselling practitioners. This should ideally be formalised in training standards and agreements with placement or clinical training providers. The learning outcome is explicit as opposed to being implicit in the training curriculum. The recommendation here is one which training providers need to provide considerable attention to. See Vignette 4 (below) for further elaboration of this theme.

\section{Evaluation of the training competencies achieved by student practitioners in the field.}

How might we begin to achieve excellence in the development of enabling environments in practitioner training? This article has started to highlight consideration of methods and standards in psychotherapeutic education, but the proof is only measured by the evaluation of training competencies delivered by training providers and subsequently demonstrated by student practitioners in the field. Such evaluation offers a measure of a standard of competence and can be a useful weapon for training practitioners to demonstrate efficacy. National training standards should also be encouraged to be specific on this theme. This may be part of a code of conduct for practice, outlining ethical and moral competencies for practitioners. This needs to be specific about how disability themes are addressed in practitioner training. This can only be addressed by the inclusion of specified learning outcomes linked to such competencies.

\section{Contribute towards the development of disability research themes in professional practice.}

A significant factor in the development of enabling environments in practitioner training is clearly the development of a training environment which promotes the opportunity for students to contribute towards the development of disability research themes in professional practice. There is clearly a parallel process between this ideal and the promotion of disability themes in curriculum delivery. The author's position in response to this recommendation is that continuing professional development frameworks for psychotherapeutic educators should ideally incorporate disability training to highlight contemporary research agendas. This can be achieved by incorporating current research themes within curriculum delivery in order to accentuate the significance of practitioner competencies in this arena. 


\section{The development of institutional policies and procedures to reflect the professional standards of the profession.}

Finally, and perhaps most significantly, the development of institutional policies and procedures need to reflect the professional standards of the counselling profession. This is a challenging arena as the ideals set out in standards of professional competence can have distinct resource implications. However, the training provider can usefully contribute to the development of institutional policies which emphasise the enabling environments agenda. The rationale needs to be made explicit and clear in the development of institutional policy. It is worth noting, however, this can be potentially viewed as an agenda-driven activity with the aim of promoting inclusion of disability themes in practitioner training and standards of professional competence.

\section{Developing Empathic Resonance in practitioner training}

Mearns \& Thorne (2005 p.xii) discuss the significance of the development of relational depth in the psychotherapeutic context described as:

"a form of encounter in which therapist and client experience profound feelings of contact and engagement with each other, and in which the client has an opportunity to explore whatever is experienced as most fundamental to her or his existence',

Consequently, applying this concept to the development of enabling environments in practitioner training, this could be proposed by working to develop an 'empathic resonance' between trainer and student on disability themes and indeed of wider issues of equality and diversity.

The author defines empathic resonance as the affective result of a successful interaction between trainer and student, or therapist and client. It is a position, once reached, which inspires the confidence and ability in the trainee to recognise the specific needs of another individual. Although the achievement of empathic resonance is a goal which could be viewed as a building block in all psychotherapeutic interventions, when applied to the context of enabling environments this would indicate that the trainer has usefully inspired the trainee counsellor (both disabled and non-disabled trainees) to recognise the significance and awareness of disability themes in professional practice and to have the confidence to move forward into professional practice with these values strongly tuned.

\section{Vignette 4}

Paulo has just completed his Counselling training and gained useful experience of working with clients in a school-based Counselling Service. He realises, however, that one of the limitations of his training is in working with clients who have a disability. He has simply not had the chance to develop his competence in this area. He does usefully reflect on this in his Reflective Journal and it is an issue he brings to you in Supervision.

Students completing their psychotherapeutic education are commonly required to submit a reflective portfolio which outlines the range of the skill development on a particular training 
programme. In order to reflect the required range of practitioner competencies, the assessment criteria could be found to be lacking if it did not contain reference to learning outcomes which cover disability themes. In the vignette above, although Paulo has identified this limitation of his existing client experience, his training provider could have been more proactive in assisting him to develop this ability and competence by developing his skills of empathic resonance with clients who have a disability. It is also worth exploring the student's understanding of the term disability. As the WHO definition includes a much wider definition to include, for example, mental health issues, it is likely that his experiences will have included disability themes when considered from a social as opposed to medical perspective.

\section{CONCLUSION}

The rationale for exploring this theme stems from recent World Health Organisation recommendations on effective and ethical disability provision. The article calls for training providers need to consider more widely how to deliver psychological training which is sensitive to the needs of service users who have a disability. The development of enabling environments in practitioner training creates a wide range of questions for psychotherapeutic training provision. It can be viewed by some as an agenda-driven activity which has distinct resource implications. From a more positive perspective, the focus on core practitioner competencies in responding to the wide range of disability issues that professional practice can create, contributes to practitioner ability and effectiveness in subsequent client work. This not only raises the efficacy of psychotherapeutic intervention but ultimately leads to competence in supporting clients in an ethically appropriate manner. It also raises the profile of the profession. The author has argued that such competencies represent core values which training providers need to incorporate in the curriculum design of psychotherapeutic training courses.

A case is made for the development of empathic resonance in client work, a point which when reached could be used as an indicator of successful therapeutic intervention and dialogue.

Given that the stated WHO demographics indicate the worldwide prevalence of people who identify as having a disability is likely to increase in the next decade, the article makes a timely call for disability themes to be made explicit in the design and delivery of psychotherapeutic training. This necessitates consideration of practice education, the development of core competencies in training, and for educational providers to fully integrate disability themes into institutional policy and procedures. As Yamamoto comments in a review of Neubauer and Tanaka's useful contribution (2011) about equity, access and capacity that higher education is growing most rapidly in the Asia-Pacific region with the consequence that university personnel as well as policy makers are facing the difficult task of balancing quality and quantity. 
It is this tension, not solely prevalent in the Asia-Pacific region, which needs to be considered in our development of future graduates from counselling and psychotherapy programmes. This comment could not be more apt when applied to the challenge of creating enabling environments in practitioner training. 


\section{REFERENCES}

Arredondo, P., Toporek, M. S., Brown, S., Jones, J., Locke, D. C., Sanchez, J. and Stadler, H. (1996) Operationalization of the Multicultural Counseling Competencies. AMCD:

Alexandria, VA

Banting \& Kymlicka (2013) Is There Really a Retreat From Multiculturalism Policies? New Evidence from the Multiculturalism Policy Index. www. Academia.edu.uk (accessed 21 May 2013)

Johnson,R \& Haigh,C (2011), 'Social psychiatry and social policy for the $21^{\text {st }}$ century: new concepts for new need - the Enabling Environments initiative' Mental Health and Social Inclusion, Volume 15, Issue 1. pp. 17-23.

Joppke,C.(2004) The retreat from multiculturalism in the liberal state: theory and policy. The British Journal of Sociology. Volume 55: Issue 2.

McFaddon,J Ed(1993) Transcultural Counseling: Bilateral and International Perspectives. Alexandria,VA: ACA

Mearns,D. and Thorne,B. (2005) Working at Relational Depth in Counselling and Psychotherapy. London: Sage.

Montgomery,M.R.,(2013) Well-being and anxiety - counselling and psychotherapy: an exploration in the Malaysian context, Asia Pacific Journal of Counselling and Psychotherapy, 4:1, 31-43, DOI: 10.1080/21507686.2013.767848

Neubauer,D and Tanaka,Y.2011 Access, Equity, and Capacity in Asia-Pacific Higher Education. (Eds) (n) Palgrave-Macmillan.

Rix,J.,Nind,M.,Sheey,K,Simmons,K. \& Walsh,C (2010). Equality, Participation and Inclusion: Diverse Perspectives.Abingdon: Routledge.

Thompson,N.(2013) Promoting Equality: Challenging discrimination and oppression $\left(3^{\text {rd }}\right.$ Ed) Basingstoke: Palgrave Macmillan.

World Health Organisation (WHO) Report on Disability December 2011: http://www.who.int/disabilities/world_report/2011/report/en/index.html (date accessed 14 Feb 2013) 